\title{
Flow velocity patterns in the pulmonary artery and pulmonary hypertension
}

\author{
Raymond Hu, MBBS • Claude Tousignant, MD • \\ Robert Chen, MD
}

Received: 17 January 2012/ Accepted: 14 March 2012/Published online: 19 April 2012

(C) Canadian Anesthesiologists' Society 2012

\begin{abstract}
Pulmonary hypertension impacts negatively on right ventricular function; however, understanding pulmonary vasculature can be difficult. Data from invasive monitoring or traditional echocardiography may not represent the full extent of pulmonary arterial disease. An important element missing from invasive monitoring is the ability to take into account the effects of pulsatile flow; therefore, mean pressures and mean flows are employed in the calculation of pulmonary vascular resistance. Traditional echocardiography yields right ventricular systolic pressures but only in the presence of tricuspid regurgitation. In these Perioperative Cardiovascular Rounds, we show the utility of interpreting pulmonary artery (PA) pulsed-wave Doppler (PWD) and colour-flow Doppler in the assessment of the pulmonary vasculature, and we describe the physiology behind their genesis. We show these concepts in a case vignette involving a patient in a low cardiac output state after a complex re-do sternotomy. Additionally, we describe four distinct patterns of PA PWD tracings and illustrate the ability of PA PWD analysis to assess the pulmonary vasculature in both a qualitative and semi-quantitative way. In the critical care setting, it is vital to understand alterations in the pulmonary circulation, and
\end{abstract}

Author contributions Raymond $\mathrm{Hu}$, Claude Tousignant, and Robert Chen were involved in manuscript preparation, and Claude Tousignant and Robert Chen were involved in manuscript review. Raymond $\mathrm{Hu}$ dealt with data collection and review of the literature.

Electronic supplementary material The online version of this article (doi:10.1007/s12630-012-9700-1) contains supplementary material, which is available to authorized users.

R. Hu, MBBS $(\bowtie) \cdot$ C. Tousignant, MD $\cdot$ R. Chen, MD

Department of Anesthesia, St Michael's Hospital, University of

Toronto, 30 Bond Street, Toronto, ON M5B 1W8, Canada

e-mail:r.hu@mail.com analysis of PA PWD can provide additional information to complement data from other sources.

\section{Clinical scenario}

Institutional Review Board approval and the patient's written informed consent were obtained for publication of this case. A 70-yr-old male presented for his third mitral valve (MV) replacement due to infective endocarditis. He had partial dehiscence of his mechanical MV and severe mitral regurgitation. He had normal coronaries, preserved left ventricular (LV) function, mild to moderate right ventricular (RV) dysfunction, and an estimated RV systolic pressure of $78 \mathrm{mmHg}$. His early postoperative course was complicated by a progressively worsening low cardiac output state, hypotension, and significant support, including norepinephrine, dobutamine, and vasopressin, to achieve a cardiac index of $1.66 \mathrm{~L} \cdot \mathrm{min}^{-1} \cdot \mathrm{m}^{-2}$ with a heart rate of 60 beats $\cdot \min ^{-1}$ and a blood pressure of $110 / 55$. His central venous pressure was $20 \mathrm{mmHg}$ and the pulmonary artery pressure (PAP) measured $45 / 23 \mathrm{mmHg}$. A wedge pressure could not be obtained, hence pulmonary vascular resistance (PVR) was not calculated.

A postoperative transesophageal echocardiogram excluded abnormalities in LV and valvular function; however, RV function was now moderately-to-severely compromised. Considerable alterations in pulmonary vascular physiology were entertained as a cause of our patient's low output state despite only modestly elevated PAP. This explanation was likely, given his preoperative long-standing left-sided valvular disease, prolonged cardiopulmonary bypass, and possible cardioprotectionrelated events. Supportive evidence of elevated pulmonary 
impedance and/or decreased compliance was found in PWD analysis of the pulmonary artery obtained from the upper esophageal short-axis view of the ascending aorta (Fig. 1) (Video available as Electronic Supplemental Material). This view showed a highly abnormal pattern of flow with apparent flow reversal during systole.

To address the alterations in the pulmonary vasculature and the low output state, pulmonary vasodilators were introduced and our patient's heartbeat was paced at a faster rate. The patient slowly improved and his trachea was extubated $86 \mathrm{hr}$ after his operation. On discharge from the intensive care unit, his systolic PAP returned to approximately $80 \mathrm{mmHg}$.

\section{Hemodynamic interpretation}

The cause of this patient's elevated PAP (left-sided valvular disease) resolved after surgery, as shown by satisfactory LV and prosthetic MV function. However, he experienced new RV dysfunction requiring considerable inotropic and pressor support although his PAP was only mildly elevated. Surrogate evidence of altered pulmonary physiology was obtained through PWD analysis of the main pulmonary artery. To understand the significance of this patient's PA PWD profile, it is necessary to understand the distinct patterns that can be found in both normal and abnormal states.

\section{Normal PA PWD pattern}

The right ventricle normally ejects with ease against a low afterload and, unlike the left ventricle, ejects despite declining and equalized RV-PA pressures (the hangout interval). This is supported by the high compliance and low resistance of the pulmonary vasculature. Accordingly, the contour of a normal pulmonary artery flow velocity (as determined by PWD) is domelike with a peak velocity (and peak pressure) occurring in mid systole ${ }^{1}$ (Fig. 2A).

\section{Abnormal PA PWD patterns}

With elevated PA pressures, the shape of the PA PWD velocity profile changes (Fig. 2 B,C,D). A major contributor to these Doppler changes is the phenomenon of "wave reflection". This refers to the returned pressure and flow waves from a pulsatile system in a semi-rigid/rigid tube. Wave reflection is not evident in the normal pulmonary circulation but can be seen in the presence of elevated pulmonary vascular impedance and decreased compliance. The reflected pressure wave is additive to forward pressure and imposes an added pressure burden on RV ejection while the reflected flow wave causes a corresponding

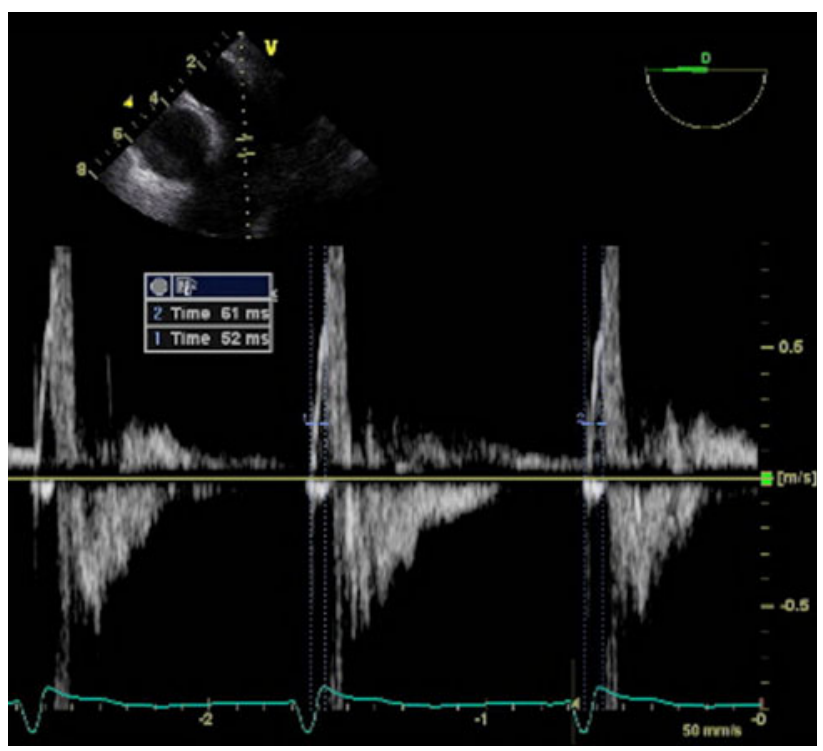

Fig. 1 Upper esophageal view of the ascending aorta in short axis at $0^{\circ}$. Pulsed-wave Doppler velocity profile with sample volume just proximal to the right pulmonary artery. The forward flow is arrested quickly. The precise acceleration time (time from onset of flow to peak velocity) is difficult to assess due to a band of aliased velocity from vortex formation. Shown here, it measures approximately 57 msec, with a value $<103 \mathrm{msec}$ having $95 \%$ sensitivity and $74 \%$ specificity in identifying patients with pulmonary vascular resistance of $>3$ WU. $^{1}$ There is early reversal of flow which continues into diastole

reduction in flow velocity. This reduction in flow velocity changes the normal domelike contour. ${ }^{2}$

Several patterns of abnormal PA PWD profiles have been described early to mid-systolic flow, including, triangular, notched, and reversed., ${ }^{3,4}$ The triangular (Fig. 2B) and notched (Fig. 2C) patterns have been attributed to wave reflection ${ }^{1}$ but do not necessarily represent degrees of severity. They represent different magnitudes and timing of the reflected wave and may also be related to stroke volume and heart rate. ${ }^{3}$ On the other hand, apparent flow reversal, as seen in our case (Fig. 2D) (Video available as Electronic Supplemental Material), occurs principally in the right posterior part of the main PA and represents the returning limb of a flow vortex rather than wave reflection. ${ }^{5}$ The formation of a vortex is not necessarily related to elevated pulmonary vascular impedance, instead, it is thought to represent biomechanical adaptation to chronic pulmonary hypertension where proximal vessels have dilated and lost their compliance. ${ }^{4,5}$

\section{Comparison of PA PWD patterns with the PA catheter}

Traditional pulmonary artery catheter (PAC)-derived data do not take into account variable flow and only assess static measures, such as resistance, rather than impedance. On the contrary, pulmonary artery PWD patterns represent 
Fig. 2 Schematic diagram and examples of pulmonary flow in normal states (A) and in pulmonary hypertension of varying severity (B) (C) (D). Notice: the rounded full contour with a central peak velocity in (A). In (B), the pattern appears more triangular and shows an earlier peak velocity. In (C), the pattern is notched. In (D), the pattern shows an abrupt and early arrest of flow velocity and flow reversal. See text for details
A

B

C

D

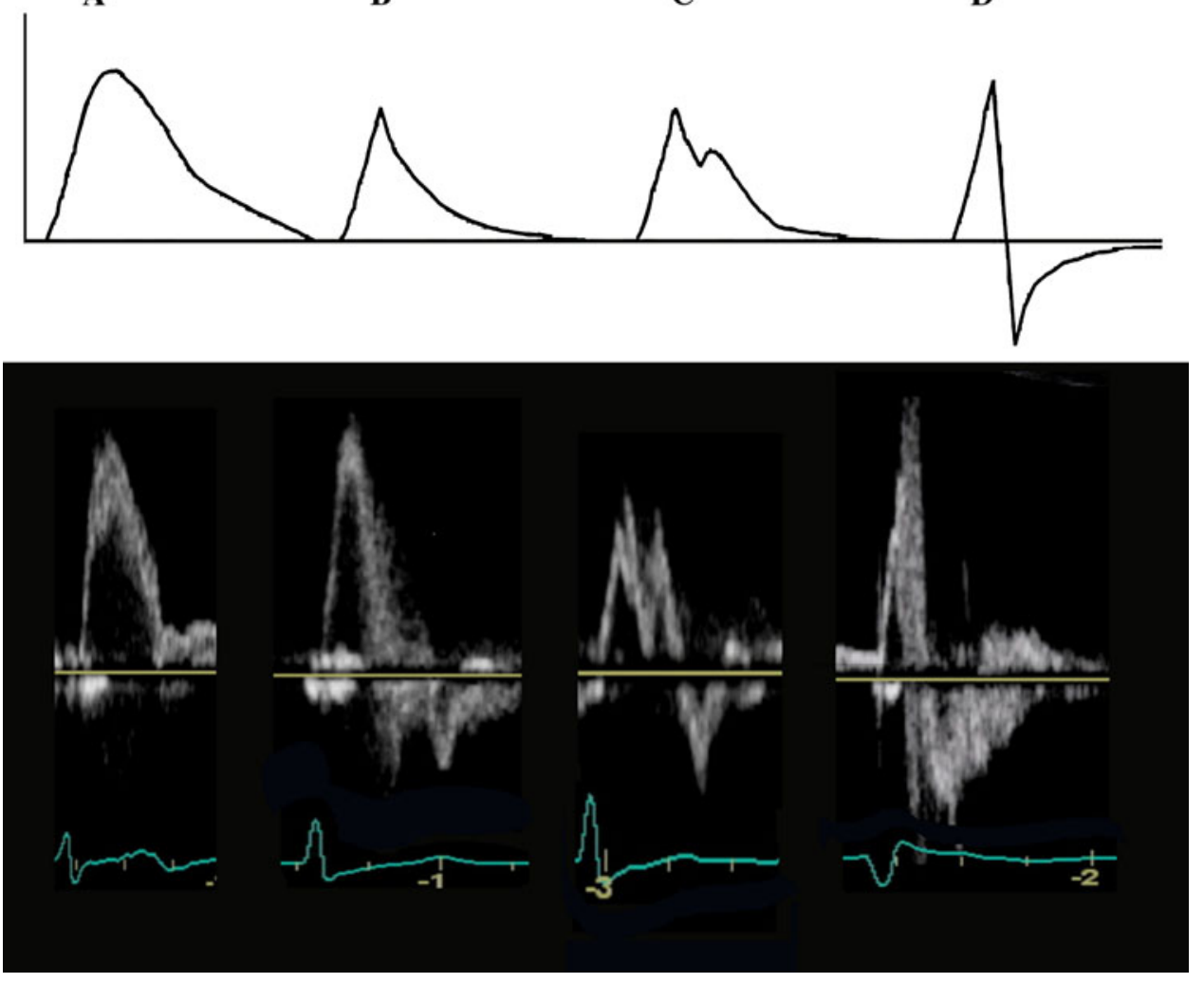

changes in a pulsatile system and thus provide more information on true hydraulic loading conditions. This is because the patterns are a result of the influence of impedance, compliance, and wave reflection, ${ }^{3}$ all of which change with pulsatile flow.

Pulmonary artery PWD patterns may thus be seen to provide a qualitative understanding of dynamic loading factors, whereas the PAC can be used to quantitate other measurements, such as PVR. This broad generalization needs to be qualified, however, with the understanding that PA PWD patterns have been used in semi-quantitative ways. In particular, the PA PWD can be used to derive the acceleration time (AcT), which is the time interval from onset to peak velocity. Acceleration time is shortened in all three abnormal Doppler patterns of pulmonary hypertension (see Figs 1 and 2) and has been shown to have a strong negative correlation with PAP. Additionally, AcT has also been correlated directly with $\mathrm{PVR}^{1}$ (see Fig. 1). Thus, this single measurement (the AcT) provides useful insight into the overall state of the pulmonary vasculature.

\section{Application}

The PA velocity profile in this patient was profoundly altered, showing a short AcT and vortex formation. This supported the interpretation of elevated pulmonary vascular impedance and/or reduced compliance and implied that these changes were a result of chronic adaptation to pathophysiology. The patient's later increases in PAP were a response to his improvement in $\mathrm{RV}$ function and stroke volume and not a failure of therapy.

\section{Conclusion}

Determining the PA velocity profile in critically ill cardiac surgical patients provides value-added information more specifically on pulmonary hydraulic load. This knowledge is complementary to the information on PVR and PAP derived from the PA catheter.

Financial support None.

Conflicts of interests None declared.

\section{References}

1. Bech-Hanssen O, Lindgren F, Selimovic N, Rundqvist B. Echocardiography can identify patients with increased pulmonary vascular resistance by assessing pressure reflection in the pulmonary circulation. Circ Cardiovasc Imaging 2010; 3: 424-32.

2. Naeije $R$, Huez S. Reflections on wave reflections in chronic thromboembolic pulmonary hypertension. Eur Heart J 2007; 28 : 785-7. 
3. Naeije R, Torbicki A. More on the noninvasive diagnosis of pulmonary hypertension: Doppler echocardiography revisited. Eur Respir J 1995; 8: 1445-9.

4. Okamoto M, Miyatake K, Kinoshita N, Sakakibara H, Nimura Y. Analysis of blood flow in pulmonary hypertension with the pulsed Doppler flowmeter combined with cross sectional echocardiography. Br Heart J 1984; 51: 407-15.
5. Reiter $G$, Reiter $U$, Kovacs $G$, et al. Magnetic resonance-derived 3-dimensional blood flow patterns in the main pulmonary artery as a marker of pulmonary hypertension and a measure of elevated mean pulmonary arterial pressure. Circ Cardiovasc Imaging 2008; 1: 23-30. 